\title{
A CMOS Based Polysilicon Nanowire Biosensor for Monitoring the Cardiovascular Disease Markers in Human Serum
}

\author{
I-Shun Wang ${ }^{1}$, Jen-Kuang Lee ${ }^{2,3,4,5}$,Chien-Chieh Peng ${ }^{1}$, Hann-Huei Tsai ${ }^{6}$, Hsin-Hao Liao ${ }^{6}$ \\ and Chih-Ting Lin ${ }^{1,2}$ \\ ${ }^{1}$ Graduate Institute of Electronics Engineering, National Taiwan University, Taipei, Taiwan \\ timlin@ntu.edu.tw. \\ ${ }^{2}$ Graduate Institute of Biomedical Electronics and Bioinformatics, National Taiwan University, Taiwan \\ ${ }^{3}$ Division of Cardiology, Department of Internal Medicine, National Taiwan University Hospital, Taiwan \\ ${ }^{4}$ Telehealth Center, National Taiwan University Hospital, Taiwan \\ ${ }^{5}$ Department of Laboratory Medicine, National Taiwan University Hospital, Taipei, Taiwan \\ ${ }^{6}$ National Chip Implementation Center, National Applied Research Laboratories, Hsinchu, Taiwan
}

\begin{abstract}
This study demonstrates a polysilicon nanowire (poly-Si NW) biosensor platform is suitable for biomolecular analytes detection in human serum. Three biomarkers, such as cardiac-specific troponin-I (cTnl), N-terminal prohormone brain natriuretic peptide (NT-proBNP) and Interleukin 6 (IL-6), for cardiovascular diseases are examined. Compared with conventional examination methods, using polysilicon nanowire biosensor not only reduces the inspection time but also economizes the cost of diagnoses. Moreover, these biosensor platforms are made by a standard complementary metal-oxidesemiconductor (CMOS) process. Based on this technology, the developed devices can be easily integrated with different functional modules, such wireless and microfluidic systems. Therefore, this work presents a good potential of CMOS based biosensor platform to accomplish the need of early diagnosis and point-of-care testing (POCT) system.
\end{abstract}

Key words: polysilicon nanowires, cardiovascular diseases, human serum, early diagnosis and pointof-care testing.

\section{Introduction}

The average life expectancy of human beings has been increased thanks to technologies and medical standards being continuously improved. However, it is inevitable that more and more people were suffered from modern civilized illness, such as cardiovascular diseases, diabetes and cancer, when entering an aging society. In general, cardiovascular diseases and coronary artery atherosclerosis which cause thousands of deaths every year are highly related. There are three important stages in coronary artery atherosclerosis, i.e. inflammation, myocardial infarction, and heart failure. There are several useful biomarkers help clinical health professionals to identify each stage, inflammation, myocardial infarction, and heart failure. Moreover, cardiovascular diseases are not only the leading cause of death for people but also costing a huge amount of money [1].

Therefore, requirements of improving healthcare services have become important issue. To accomplish these needs, present medical equipment suffers from constrains, such as bulky size, complicated operation, high cost, and long detection time. To overcome these obstacles, it is necessary to develop biosensors for easy and simple usages [2]. To address this, in this study, a poly-Si NW based CMOS biosensor chip is designed and fabricated by a standard $0.35 \mu \mathrm{m}$ CMOS process. To evaluate the sensing performance in serum samples, three biomarkers related to cardiovascular diseases are chosen as the detection targets. The results demonstrate a promising potential to perform a robust POCT device for personalized biomarkers diagnosis in human serum test. 


\section{Materials and Methods}

To accomplish the purpose of low-cost and mass production, poly-Si NW biosensors were manufactured by a $0.35 \mu \mathrm{m} \quad 2 \mathrm{P} 4 \mathrm{M}$ commercially-available CMOS fabrication technology. The detail poly-Si NW design was described on our previous work [3]. There were four poly-Si NWs to form a Whetstone bridge architecture. Two of poly-Si NWs were exposed after post-etching processes, as shown in Figure 1. Next, as shown in Figure 2, the implemented biosensor chip was bonded on a printed-circuit-board with a plastic reservoir filled with PBS buffer solution as operated under bio-related experiments. A conventional surface functionalized processes was introduced to functionalize the exposed poly-Si NWs in order to immobilize the specific antibody for the target protein. Figure 3 shows the process flow of process immobilization. After the poly-Si NWs were treated in $2 \%$ APTES alcohol solution for $1 \mathrm{~h}$ for silanization, $2.5 \%$ glutaraldehyde (GA) solution was dripped on exposed poly-Si NWs for $1 \mathrm{~h}$ in room temperature. Finally, antibodies for each biomarker were dripped on the membrane and samples were stored in fridge at $4 \mathrm{oC}$ overnight. Before measurement, bovine serum albumin with phosphate buffered saline (PBS) buffer solution was applied on the poly-Si NWs surface to block uncross-linked binding site. Then, the chip was ready for measurements after rinsed with PBS buffer.

In this research, all of the liquid samples were about $200 \mu \mathrm{l}$ in volume and exchanged by pipetting. It should be noted that each experimental result was measured from 3 independent chips because of the limited amount of chips. To obtain the output response of poly-Si NW biosensors, Keithley 6485 picoammeter was used. The experimental protocol could be briefly described as follows: (a) Inject $0.01 \mathrm{x}$ PBS buffer solution for 5 minutes to obtain an initial reference base line; (b) Inject and incubate a specific concentration of testing target biomarker; (c) The poly-Si NWs were rinsed off three times by using $0.01 x$ PBS buffer to remove unbinding biomarkers; (d) The output voltage was measured with $0.01 \times$ PBS buffer. All of detection results of the biomarkers both in commercial target proteins and the clinical human serum samples were calculated follow an equation which was defined as normalized detection response (NDR):

$$
\left(\Delta V / V_{0}\right)=\left(\left|V_{S}-V_{0}\right| / V_{0}\right)
$$

Where $V_{0}$ is the output voltage measured from the initial background level in the PBS buffer, $V_{S}$ is the output voltage detected in the measurement buffer after the biomolecular binding process [4].

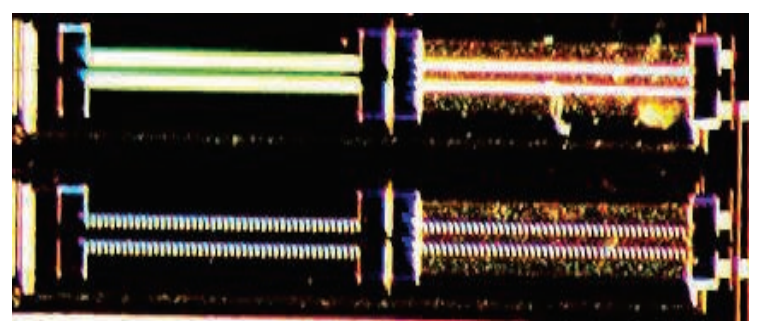

Fig. 1. Picture of Whetstone bridge architecture form Poly-Si NW biosensor chip.

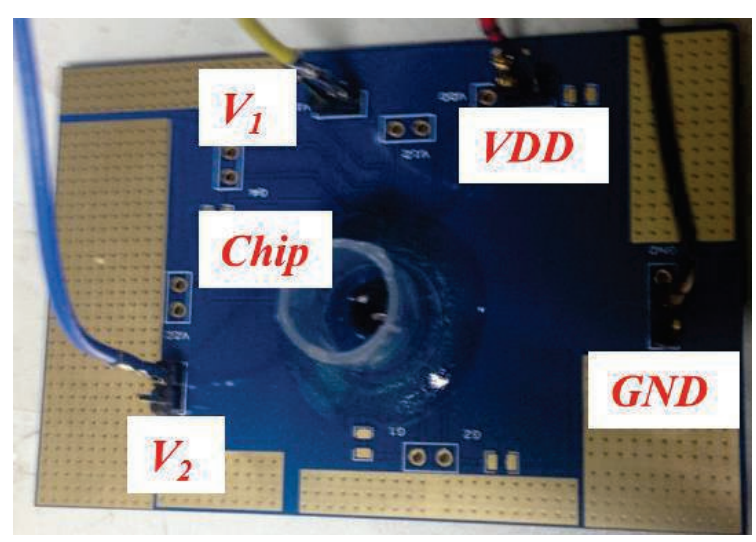

Fig. 2. Picture of measurement setup. Poly-Si NW biosensor chip was bond on printed-circuit-board.

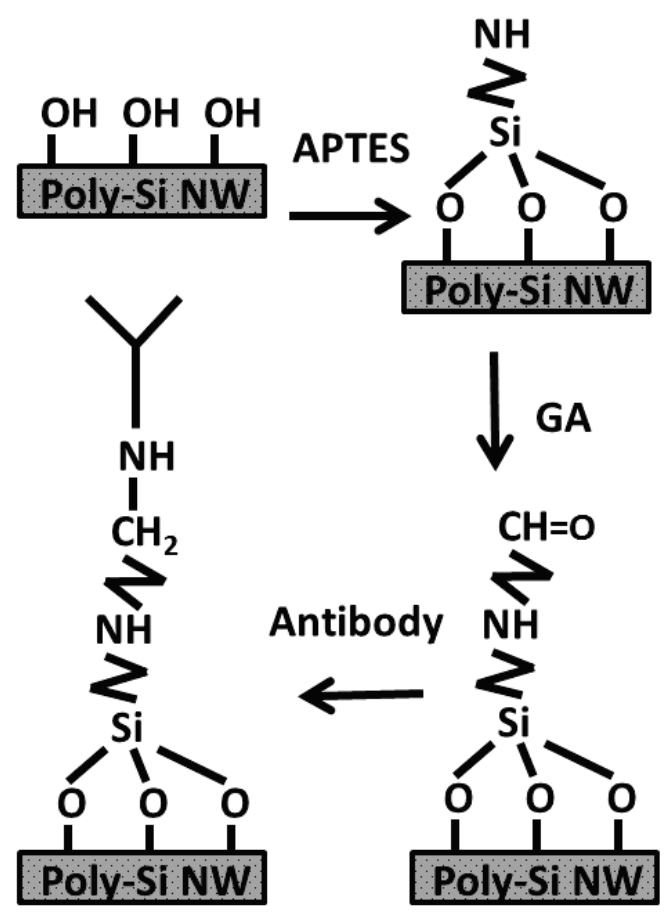

Fig. 3. Schematic of Antibody immobilization process flow 


\section{Results and Discussion}

It is important to validate the poly-Si NWs by measuring artificial protein in the first step. The isoelectric point $(\mathrm{pl})$ values of these three target proteins and the $\mathrm{pH}$ value of the measurement buffer dominate the amount of charge. According to previous reports, the pl value of IL-6, cTnl and NT-proBNP are generally 6.2, 5.2-5.4 and 9.9 [5]. Therefore, in order to obtain the evident signal responses, the $\mathrm{pH}$ value of measurement buffer in IL-6 detection was controlled at 8.0. Figure 4 shows the measurement result of IL- 6 detection. When the concentration of target protein was larger, the higher output signal response was observed.

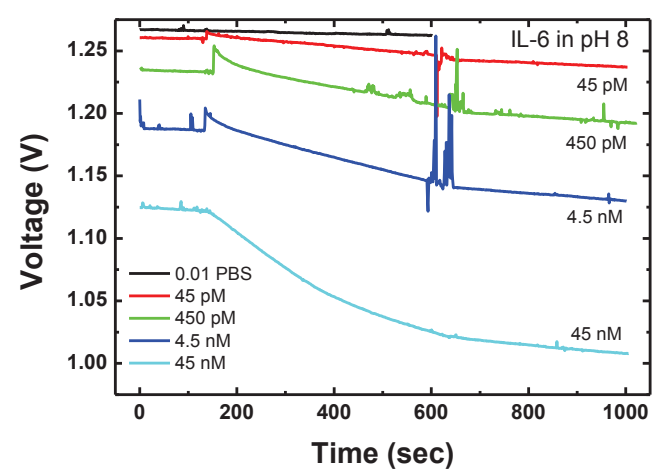

Fig. 4. An experimental result of the functionalized poly-Si NWs biosensor chip measuring artificial IL-6 protein in $0.01 X P B S$.

On other hand, the $\mathrm{pH}$ value of measurement buffer in cTnl and NT-proBNP detection was controlled at 7.4 because of the obvious difference in its $\mathrm{PI}$ values and buffer's $\mathrm{pH}$ value. Figure 5 (a) to (c) shows the biosensing results of cTnI, NT-proBNP and IL-6 in 0.01X PBS, respectively. The lowest concentration of each biomarker represents the limit of detection (LOD) from a sensor response that was equal to three times the standard deviation of the baseline noise.

After investigated on commercial protein of this three cardiovascular disease biomarkers, clinical human serum with target proteins were examined. We used the health people's serum as a control sample in this study. On other hand, three concentrations were chosen for each of the cardiovascular disease biomarker. Before using those serum samples in this study, all of the human serum samples were examined by central laboratory in National Taiwan University Hospital. Detail concentration of three cardiovascular disease related biomarkers are shown in Table 1. The measurement results of three biomakers in human serum could be discriminated easily as shown in Fig. 6 (a) to Fig.6 (c). The different outcomes between different proteins are caused by the different target concentrations and the isoelectric point value issue [6-7]. Based on this work, it is promising to employ the developed CMOS based poly-Si NW biosensor platform for POCT system.
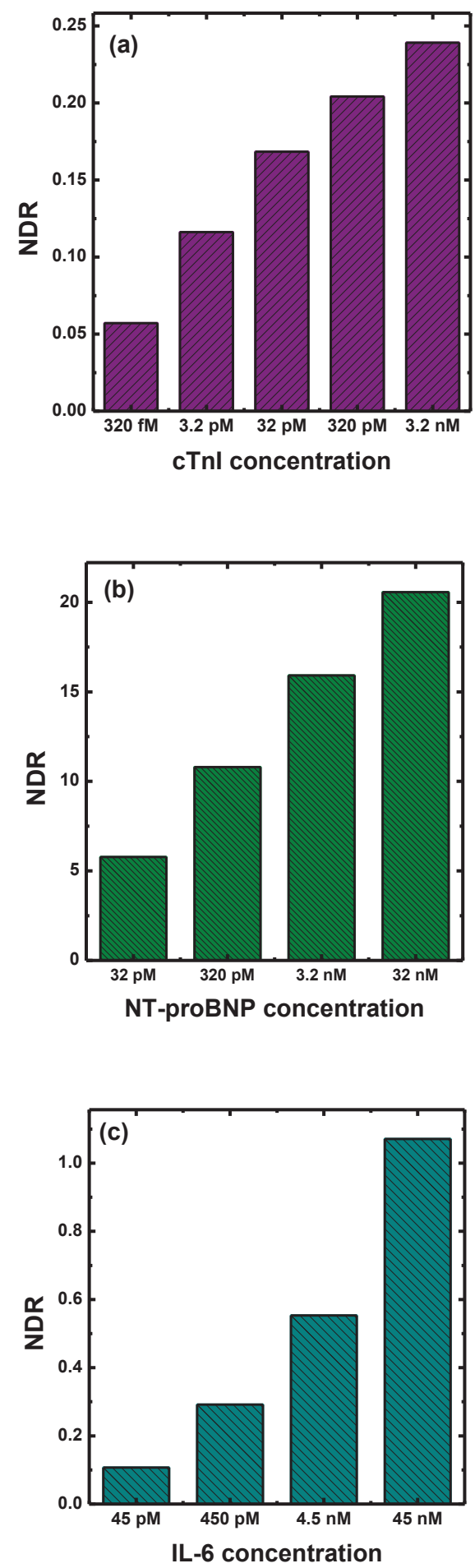

Fig. 5. The experimental responses of the functionalized poly-Si NWs biosensor chip measuring artificial biomarkers, (a) cTni, (b) NT-proBNP and (c) IL-6, in 0.01X PBS. 

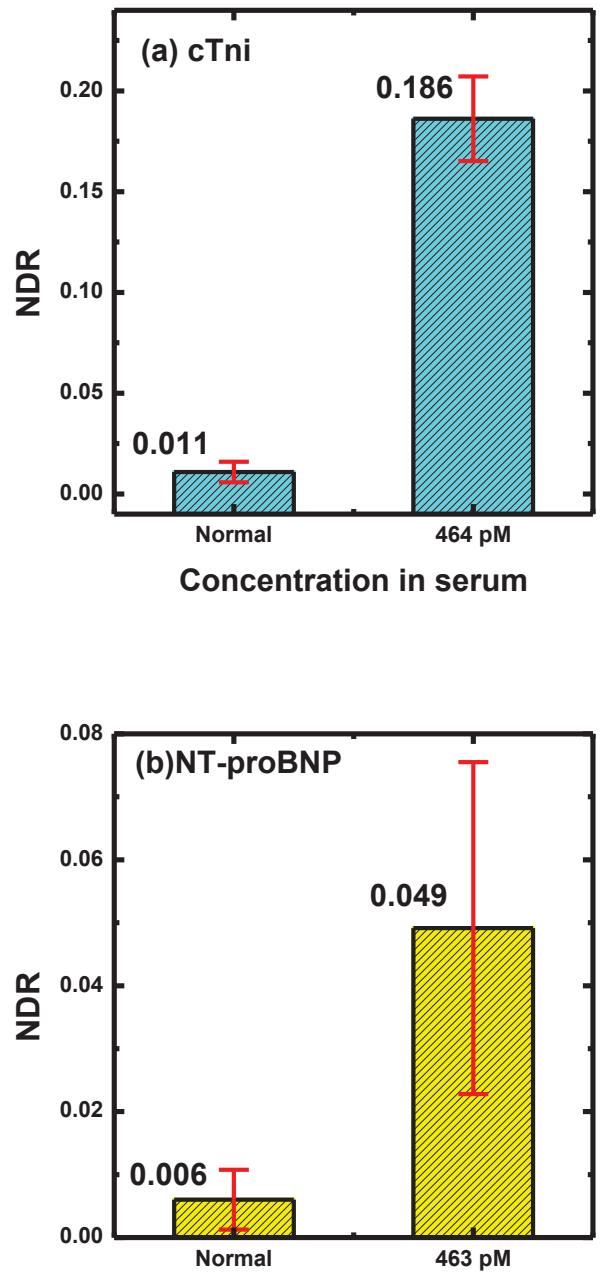

Concentration in serum

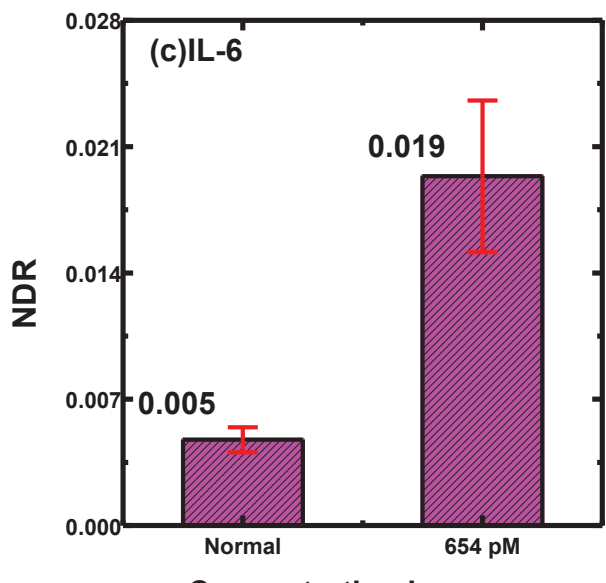

Concentration in serum

Fig. 6. The experimental responses of the functionalized poly-Si NWs biosensor chip measuring clinical human serum samples with and without three biomarkers, (a) cTni, (b) NT-proBNP and (c) IL-6, related to cardiovascular diseases.
Tab. 1: Detail experiment parameters of three biological indicators related to cardiovascular diseases, cTni, NT-proBNP and IL-6, in this research.

\begin{tabular}{cccc}
\hline \hline $\begin{array}{c}\text { biological } \\
\text { markers }\end{array}$ & cTnl & NT-proBNP & IL-6 \\
\hline $\begin{array}{c}\text { clinical } \\
\text { sample } \\
\text { concentration } \\
\text { (pM) }\end{array}$ & 464 & 463 & 654 \\
\hline $\begin{array}{c}\text { isoelectric } \\
\text { point value }\end{array}$ & 5.4 & 9.9 & 6.2 \\
\hline $\begin{array}{c}\mathrm{pH} \text { value of } \\
\text { measurement } \\
\text { buffer }\end{array}$ & 7.4 & 7.4 & 8.0 \\
\hline \hline
\end{tabular}

\section{Conslusion}

In this work, we present a poly-Si NW biosensor platform is suitable for biomolecular analytes detection for cardiovascular diseases. Three important target makers, i.e. cTnl, NT-proBNP and IL-6, are detected not only in artificial protein but also in clinical samples successfully.

\section{References}

[1] P.A Heidenreich et al, Forecasting the future of cardiovascular disease in the United States: A policy statement from the American Heart Association, Circulation 123, 933-944 (2011); doi: 10.1161/CIR.0b013e31820a55f5

[2] Q. Mei and Z. Zhang Photoluminescent graphene oxide ink to print sensors onto microporous membranes for versatile visualization bioassays, Angewandte Chemie 51, 5602-5606 (2012); doi: 10.1002/anie.201201389

[3] C.-W. Huang et.al, A CMOS wireless biomolecular sensing system-on-chip based on polysilicon nanowire technology, Lab on a Chip 13, 4451-4459 (2013); doi: 10.1039/c3lc50798j

[4] P.-W. Yen et al, A device design of an integrated CMOS poly-silicon biosensor-on-chip to enhance performance of biomolecular analytes in serum samples, Biosensors and Bioelectronics 61, 112118 (2014); doi: 10.1016/j.bios.2014.05.010

[5] I. Lee et al, Detection of cardiac biomarkers using single polyaniline nanowire-based conductometric biosensors, Biosensors and Bioelectronics 2, 205-220 (2012); doi : 10.3390/bios2020205

[6] H.-L Gao et.al, A nanochannel array based device for determination of the isoelectric point of confined proteins, Phys. Chem. Chem. Phys., 14 (2012) 9460-9467.

[7] Protein calculator v3.3 http://www.scripps.edu/ cdputnam/protcalc.html 